\title{
Le loup, la métairie et la forge : dialogue entre un historien et un écologue
}

\author{
Éric Fabre ${ }^{1}$, Jean Cantelaube ${ }^{2}$ \\ 1 Écologue et historien, IUT de l'Université de Provence, 04000 Digne-les-Bains, France et UMR6583 Centre de recherche d'histoire \\ quantitative de l'Université de Caen-Basse-Normandie, 14032 Caen cedex 5, France \\ 2 Historien, UMR5136 FRAMESPA, Université Toulouse-Le-Mirail, 31058 Toulouse cedex 9, France
}

\section{Introduction}

Le loup, la métairie et la forge à la catalane : au cours de nos travaux, en dépit de la diversité des objets observés, nous avons, inévitablement, rencontré l'environnement des sociétés anciennes sur lesquelles nous enquêtions. Or, dans tous les cas, il est apparu que «la nature » avait un rôle-clé dans l'évolution historique, qu'elle était un facteur inévitable à la fois pour décrire et pour comprendre. Certes, cette constatation est d'une grande banalité. Cependant, de maladresses en tâtonnements et en incertitudes, une prise de conscience s'est peu à peu imposée : il est nécessaire pour conserver une démarche rigoureuse de se tourner vers les sciences biologiques. Les regards ont alors cherché à se croiser.

Jean Cantelaube, historien des techniques, participait en effet aux travaux d'une équipe de biogéographes et de biologistes qui étudiaient le paysage pyrénéen et cherchaient à connaître l'impact humain sur la forêt. Le constat que dégagent ces travaux est unilatéral : les hommes, par leur activité industrielle, ici sidérurgique, modifient le milieu et composent une forêt nouvelle. En revanche, les recherches de Jean Cantelaube, conduites dans la tradition de l'histoire des techniques, mettaient

Auteur correspondant:

É. Fabre, Eric.Fabre@univ-provence.fr

Jean Cantelaube est historien des techniques. Ses travaux portent sur la métallurgie et, notamment, sur son rôle dans la construction du paysage pyrénéen. Éric Fabre, maître de conférences en écologie et chercheur en histoire, tente d'interpréter des dynamiques écologiques (grands prédateurs, masses forestières) par des processus historiques, donc humains. en évidence que les changements du milieu, loin d'être toujours les conséquences des changements techniques, peuvent aussi en être les causes. Les réflexions nées de cette constatation, et après une recherche historiographique poussée, l'ont conduit à se demander si l'écologie n'apporterait pas des réponses pertinentes à ses interrogations.

Le questionnement d'Éric Fabre était initialement celui d'un biologiste. Il portait en particulier sur la dynamique de la biodiversité et le fonctionnement des écosystèmes forestiers. S'il était évident qu'un recul temporel était nécessaire pour comprendre la dynamique du loup ou les processus de mise en place de la forêt, il avait dépassé ce constat en développant une réflexion à partir de ses compétences d'historien acquises par un double cursus universitaire.

Ensuite, nos rencontres ont permis que nos idées se confrontent et s'enrichissent. Une question récurrente de nos débats est celle de l'efficacité potentielle de l'écologie pour l'historien : offre-t-elle un outillage conceptuel mobilisable dans un cadre historique? Nous ne prétendons pas donner ici une réponse absolue à cette question, mais seulement transmettre le témoignage de notre expérience concrète de chercheur. Il ne s'agit donc pas ici de présenter un bilan des nombreuses démarches interdisciplinaires ayant associé des historiens, des archéologues et des écologues en France et dans le monde pour une intégration des dynamiques écologiques et sociales, mais de proposer, par un retour sur nos recherches, quelques remarques préliminaires à propos des difficultés rencontrées, prolégomènes à un dialogue interdisciplinaire aux contours encore flous. Dialogue 
stimulé par le fait que l'un d'entre nous est à la fois écologue et historien.

L'un des tout premiers problèmes qui se pose est celui du langage. Comment l'historien puise-t-il dans le lexique du biologiste et, surtout, quelles sont les déformations que subissent alors les concepts? On voit la diversité et la complexité des questions soulevées.

\section{Le loup ou la biologie ignorée}

Le loup, par sa charge symbolique, est un des rares animaux à bénéficier d'un regard privilégié en sciences humaines, et l'histoire n'est pas en reste. Si, dans une démarche ethnologique ou anthropologique, il est une porte d'entrée explicite vers les sociétés humaines ${ }^{1}$, des travaux de géographie empruntent le même chemin pour décrire les jeux d'acteurs autour de l'idée d'environnement $^{2}$. À la suite de Robert Delort ${ }^{3}$, la bibliographie de $l^{\prime}$ histoire des animaux s'est considérablement étoffée ${ }^{4}$, et il n'est pas dans notre propos de l'aborder autrement que sous l'angle de la position du loup dans le champ d'analyse de l'historien. C'est rarement l'histoire propre des «bêtes » qui est écrite, mais plutôt celle de la mainmise des hommes sur elles, que ce soit par la chasse, par utilitarisme (élevage, jeux du cirque, etc.), par salutaire nécessité (réduction de nuisance) ou par curiosité (parcs zoologiques et découvertes scientifiques). Dans tous les cas, et c'est bien normal sous l'égide d'une science humaine quelle qu'elle soit, le milieu de vie des animaux n'est pas analysé à l'aune des besoins de l'espèce mais au travers du seul regard humain.

De façon récente, Jean-Marc Moriceau intègre l'analyse du domaine vital de meutes de loups anthropophages pour renforcer l'idée que les auteurs des attaques sont bien des loups et non des chiens errants ou

\footnotetext{
1 Bobbée, S., 2002. L'Ours et le loup. Essai d'anthropologie symbolique, Paris, Éditions de la MSH/Inra ; Mauz, I., 2002. Gens, cornes et crocs. Relations hommes-animaux et conceptions du monde, en Vanoise, au moment de l'arrivée des loups. Doctorat en sciences de l'environnement, Cemagref, ENGREF.

2 Benhammou, F., 2003. Les grands prédateurs contre l'environnement? Faux enjeux pastoraux et débat sur l'aménagement des territoires de montagne, Le Courrier de l'Environnement de l'INRA, 48, 5-12 ; Benhammou, F., Baillon, J., Senotier, J.-L. (Eds), 2004. La Cohabitation hommes-grands prédateurs en France (loup et ours). Actes du colloque des 21 et 22 mars 2004, Muséum d'Orléans, Recherches Naturalistes en Région Centre, 14.

${ }^{3}$ Si Robert Delort n'est pas le seul historien à faire cas des animaux, il est le premier à focaliser sur eux le regard de l'historien en rassemblant une documentation éparse, bien souvent issue de champs disciplinaires ignorés des sciences humaines.

4 Pour une synthèse : Baratay, E., Mayaud, J.-L., 1997. L'histoire de l'animal, bibliographie, Cahiers d'histoire, 47, 443-480. Elle est parfois l'œuvre de géographes : De Planhol, X., 2004. Le Paysage animal. L'homme et la grande faune : une zoogéographie historique, Paris, Fayard.
}

même des hommes ${ }^{5}$. Ici, le but est d'utiliser un paramètre de la biologie des loups pour mieux appuyer l'argumentation relative à l'appartenance taxonomique des individus impliqués dans les agressions sur les hommes.

Nous pensons qu'il serait souhaitable que cette prise en compte de la biologie de l'espèce dans les travaux d'histoire de l'animal se généralise. Ceux-ci seraient alors plus fréquemment remis dans leur contexte environnemental, et pas seulement social ou culturel. Dans le cas du loup, une démarche d'écologie historique a été initiée ${ }^{6}$; il faut savoir que la plupart des travaux concernant cet animal relèvent de l'histoire culturelle, c'est-à-dire qu'on ne le considère qu'à travers ce que l'Homme en pense. On ne cherche donc pas à connaître la « vérité » de l'animal hors de sa perception par les hommes, voire de la symbolique qu'ils lui attribuent. Par écologie historique, nous entendons donc un retour à l'animal par la réalisation d'une véritable écologie de l'espèce, dans la limite des sources d'archives qui la permettent.

Outre l'intérêt théorique de ce questionnement, la richesse générée par le croisement des regards a, dans le cas du loup pris ici en exemple, un intérêt pratique pour les gestionnaires qui doivent faire face à son retour. Cette dynamique est la conséquence d'un changement de statut d'espèce nuisible à espèce protégée, mais aussi, et peut-être surtout, du changement des espaces ruraux où la couverture forestière s'étend : des milieux qui lui sont favorables se développent et sont prêts à l'accueillir ${ }^{7}$. Les mutations technico-économiques et sociales tiennent donc le premier rôle dans la création des écosystèmes concernés.

\section{La métairie ou l'histoire économique et sociale en fondement des écosystèmes}

L'écologie du paysage, articulant les échelles de temps et d'espace, est conceptuellement équipée pour mobiliser le recul historique nécessaire à la compréhension des processus biologiques. Mais insistons : la prise en compte de ce temps passé, pour expliquer les situations actuelles, ne

\footnotetext{
${ }^{5}$ Moriceau, J.-M., 2007. Histoire du méchant loup : 3000 attaques sur l'homme en France, $X V^{e}-X X^{e}$ siècles, Paris, Fayard.

${ }^{6}$ Fabre, É., Alleau, J., 2007. La disparition des loups ou essai d'écologie historique. Communication à la journée d'étude "L'animal sauvage entre nuisance et patrimoine : approche socio-historique des relations homme-nature, $X V I^{\mathrm{e}}-\mathrm{XX}$ e siècles ", 4 mai, ENS Lyon.

7 Orsini, P., Fabre, É., 2006. Le loup, quelques éléments sur sa disparition de Haute-Provence au XIX ${ }^{\mathrm{e}}$ siècle, Bulletin du Comité scientifique du Parc naturel régional du Verdon, 1, 121-153 ; Fabre, É., Alleau, J., 2007. État de l'environnement et disparition du loup : approche de la relation sur le territoire de l'actuel PNR du Verdon (1750-1890). Rapport d'étude pour le Parc naturel régional du Verdon.
} 
saurait se faire hors du cadre méthodologique et conceptuel de la discipline historique. Si l'on ne s'improvise pas biologiste - et on a vu que les historiens de l'animal ne s'y essaient que trop peu - on ne devient pas plus historien par le seul fait de se pencher sur le passé. Ainsi, si un recul chronologique enrichit les travaux d'écologie du paysage, l'association des compétences disciplinaires ne suit pas toujours. Les exemples ne sont pas rares de mauvais usages, par les écologues, de sources pourtant d'un classicisme éprouvé pour l'historien.

Sur le piémont pyrénéen, la déprise humaine est importante. Les outils classiques de l'histoire économique et sociale (démographie, étude de la propriété, etc.) ont été mobilisés pour comprendre quand, pourquoi et comment certains domaines agricoles isolés ont été désertés entre le milieu du XVIII ${ }^{\mathrm{e}}$ et le milieu du XXe siècle, les terres se couvrant alors de forêts ${ }^{8}$. De nouveaux milieux se créent, très défavorables à plusieurs taxons, comme les orchidées qui disparaissent avec les prairies qui les hébergeaient, mais propices au développement d'une grande faune d'ongulés tels que cerfs, chevreuils ou sangliers. Des explications d'ordre historique de la mise en place de ce nouvel environnement ont été avancées, la forêt a été analysée dans sa nouvelle configuration ${ }^{9}$. Il convient alors de passer de la description des milieux à celle des processus écologiques qui s'y déroulent. Le fil conducteur est le suivant : après l'analyse historique de la désertion et du développement du couvert forestier, après la description de ces nouveaux milieux, le chercheur s'attache à comprendre la dynamique des grands ongulés qui colonisent les espaces qui s'offrent à eux. Si l'étude de la dynamique de ces groupes animaux et végétaux relève incontestablement de la science écologique, ce n'est que dans l'approche historique qu'elle peut trouver le fondement des mutations observées.

Une telle intégration réciproque entre milieu et activités humaines a déjà été tentée lors de l'étude de la sidérurgie pyrénéenne.

\section{La forge à la catalane ou une vision systémique de l'industrie}

La forge est "dévoreuse de forêts ». Au début du $X X^{\mathrm{e}}$ siècle, le botaniste Henri Gaussen constate la déforestation des Pyrénées, l'absence de pinèdes d'altitude, la faible altitude de la limite entre les forêts et les pâturages. Or, les conditions bioclimatiques n'expliquent

\footnotetext{
8 Fabre, É., 2008. Les Métairies en Languedoc. Désertion et création des paysages contemporains (XVIII - XIX ${ }^{e}$ siècles), Toulouse, Privat.

9 Fabre, É., Surjus, A.-L., 2008. Forêt de déprise, forêt d'emprise : colonisation forestière et maîtrise de l'espace boisé en Chalabrais (début du XIX ${ }^{\mathrm{e}}$ - fin du $\mathrm{XX}^{\mathrm{e}}$ siècle). Communication au colloque "Les paysages méridionaux », FRAMESPA et archives départementales de l'Aude, 23 et 24 mai, Carcassonne.
}

pas ces anomalies : le biologiste leur attribue une origine anthropique. Depuis, les travaux qui utilisent «la mémoire des charbonnières $»^{10}$, conduits sous la direction de Georges Bertrand, ont non seulement confirmé la responsabilité de l'homme mais précisé l'un de ses moyens $\mathrm{d}^{\prime}$ action : la sidérurgie ${ }^{11}$.

Au XVII ${ }^{e}$ siècle, la forge à la catalane naît dans un contexte de raréfaction du charbon de bois consécutif à la dégradation des forêts : elle est une réponse à cette pénurie alors que la demande de fer augmente. Avec ses innovations techniques (trompe hydro-éolienne pour la soufflerie et foyer quadrangulaire), elle se révèle capable de satisfaire l'impérieuse nécessité d'économiser le combustible tout en transformant des quantités de minerai de plus en plus grandes, c'est-à-dire en produisant plus. Cet ensemble novateur crée un savoir-faire spécifique. Puis, après sa généralisation, la recherche constante d'une moindre consommation de charbon de bois est un trait structurel fort de la sidérurgie à la catalane (amélioration du rendement de la réduction, finesse du tour de main des forgeurs) qui lui permet de conserver une place centrale dans la montagne pyrénéenne jusque dans la seconde moitié du XIX ${ }^{\mathrm{e}}$ siècle. La forge s'est adaptée aux conditions que lui imposaient le milieu et ses transformations ${ }^{12}$.

Dans le même temps, elle a imposé en retour un aménagement de la forêt dans un cadre législatif et administratif contraignant. Il s'agit d'abord de privilégier certaines essences, puis d'améliorer les pratiques culturales : les modes d'exploitation séculaires qui se développent alors, aboutissent à la généralisation des bois taillis de hêtre qui, rejetant de souche, éliminent le sapin. Le milieu forestier est donc façonné pour le besoin de la ressource industrielle.

Des logiques techniques, sociales, économiques et écologiques expliquent la pérennité et la puissance du système productif à la catalane dans une société agro-pastorale. La démarche systémique éclaire les interactions entre le milieu et la forge dans des allersretours incessants ${ }^{13}$. Un pas a été franchi : considérer la forge comme partie prenante d'un écosystème.

\footnotetext{
10 Bonhôte, J., 1998. Forges et forêts dans les Pyrénées ariégeoises. Pour une histoire de l'environnement, Aspet, Pyrégraph.

11 De 1992 à 2006, de nombreuses thèses de doctorat de l'Université de Toulouse-Le Mirail portant sur «l'histoire de l'environnement » ont été conduites.

12 Cantelaube, J., 2009. Le charbon de bois et la forge à la catalane (Pyrénées, XVII ${ }^{\mathrm{e}}$ - XIX ${ }^{\mathrm{e}}$ siècles), in Menozzi, M.-J., Flipo, F., Pécaud, D. (Eds), Énergie et Société. Sciences, gouvernances et usages, Nantes, Edisud, 35-45.

13 Cantelaube, J., 2005. La Forge à la catalane dans les Pyrénées ariégeoises, une industrie à la montagne (XVII ${ }^{e}$ XIX ${ }^{e}$ siècle), Toulouse, CNRS/Université de Toulouse-Le Mirail.
} 


\section{Le regard de l'historien fatalement biaisé ? Le poids de l'histoire de l'environnement}

Les trois études que nous venons de présenter illustrent les difficultés rencontrées pour croiser les regards. La bibliographie montre en particulier que la relation entre histoire et écologie est déséquilibrée. Nos travaux se placent dans un ensemble plus vaste : l'histoire du climat telle qu'elle est définie par Emmanuel Le Roy Ladurie $^{14}$, l'histoire de l'environnement demandée par Georges Bertrand ${ }^{15}$, l'histoire des catastrophes naturelles devenues objets d'histoire dans les années 1990. Il faut ajouter bien entendu les travaux de Robert Delort sur les animaux ${ }^{16}$, sur l'environnement en général ${ }^{17}$, sans oublier les recherches conduites aujourd'hui sur l'histoire de nombreux milieux dont une analyse des sources est donnée par Andrée Corvol ${ }^{18}$. Une présentation historiographique et une réflexion épistémologique sont conduites par Georges Pichard qui conclut sa thèse en

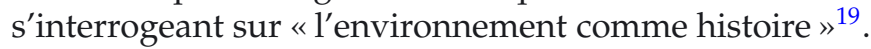
Pour lui, l'histoire "des éléments naturels » est nécessaire, mais elle doit toujours "garder le contact » avec «l'histoire humaine». Pas question de négliger l'importance et l'intérêt de ces travaux, nous nous appuyons sur eux. Cependant, leur orientation ne nous a pas permis de répondre d'une façon totalement satisfaisante aux questions que nous nous posions, laissant un goût d'inachevé!

Pour faire avancer la réflexion, il est indispensable de définir les mots utilisés par l'historien lorsqu'il prend ou veut prendre en compte «la nature » et non seulement «l'environnement ». Ils sont nombreux, rattachés à différentes disciplines : tableau géographique, cadre, conditions naturelles, données naturelles, ressources naturelles, etc. Tous induisent un déterminisme dans le choix des activités humaines. «Milieu » est déjà plus large, mais

\footnotetext{
${ }^{14}$ Le Roy Ladurie, É., 1983. Histoire du climat depuis l'an mil, Paris, Flammarion ; Le Roy Ladurie, É., 2004. Histoire humaine et comparée du climat. Vol 1, Canicules et glaciers, XIII ${ }^{e}$-XVIII ${ }^{e}$ siècles, Paris, Flammarion; Le Roy Ladurie, É., 2006. Histoire humaine et comparée du climat. Vol 2, Disettes et révolutions (1740-1860), Paris, Flammarion.

15 Bertrand, G., 1992. Pour une histoire écologique de la France rurale, in Duby, G., Wallon, A. (Eds), Histoire de la France rurale, Paris, Flammarion, tome 1, 39-118.

16 Delort, R., 1982. Les Animaux ont une histoire, Paris, Le Seuil. 17 Delort, R., Walter, F., 2001. Histoire de l'environnement européen, Paris, Presses universitaires de France.

18 Corvol, A. (Ed.), 1999. Les Sources de l'histoire de l'environnement. Le XIX ${ }^{e}$ siècle, Paris, L'Harmattan ; Corvol, A. (Ed.), 2003. Les Sources de l'histoire de l'environnement. Le XXe siècle, Paris, L'Harmattan; Abbé, J.-L., 2006. À la conquête des étangs. L'aménagement de l'espace en Languedoc méditerranéen (XII ${ }^{e}-X V^{e}$ siècle), Toulouse, Presses universitaires de Toulouse-le Mirail.

19 Pichard, G., 1999. Espaces et nature en Provence. L'environnement rural, 1540-1789. Thèse de doctorat d'histoire, Université de Provence.
}

la notion de contrainte, plus ou moins maîtrisée au cours des siècles, est toujours présente. Certains auteurs vont même jusqu'à parler de «stress écologique ${ }^{20}$. La relation à sens unique est parfaitement éclairée par l'expression : «contraintes du climat». L'étude de «l'environnement » semble plus adéquate parce qu'il est toujours celui des hommes, que l'on s'appuie sur ses définitions du domaine savant (géographie, par exemple) ou technique (ISO14000). Par conséquent, l'intégration de l'environnement dans l'histoire ne peut (ou ne devrait) pas poser de problème, car il est une composante de la vie des hommes. Or, dans la plupart des cas, seuls l'action de l'homme et son impact sont pris en considération. Les travaux oublient souvent de chercher l'effet inverse, c'està-dire celui de la nature sur l'évolution de la société. Cette dimension unidirectionnelle privilégie donc la notion de «crise écologique ».

Cet anthropocentrisme est parfaitement légitime s'il est pleinement et explicitement assumé, mais une véritable histoire de l'environnement devrait généraliser l'intégration des allers et retours entre milieu et sociétés humaines dans une approche systémique. Une telle démarche est possible et a déjà donné de beaux fruits dans le cadre de l'écologie historique.

\section{Croiser les regards, l'écologie historique. Pour dépasser l'histoire de l'environnement}

Notre réflexion porte sur des études historiques conduites par des historiens. Jusqu'ici, ce regard disciplinaire a prédominé mais il est temps de prendre en compte celui de l'écologue. L'écologie est une branche des sciences de la vie, elle est une discipline se donnant pour but d'étudier l'ensemble des relations que les populations entretiennent entre elles et avec leur milieu. Insistons sur un point : les populations ne sont pas qu'humaines. Des biologistes ont déjà utilisé des sources historiques pour prendre un recul temporel sur leur objet d'étude. L'histoire est un outil, un ensemble de méthodes qui permettent à l'écologue d'accéder à des données normalement acquises par l'expérience de laboratoire ou l'observation de terrain, mais qui sont inaccessibles quand l'écosystème étudié a disparu. C'est là que la méthode historique intervient pour alimenter la connaissance de chacun des facteurs agissant dans le milieu considéré : une écologie dans l'histoire est initiée. Nous empruntons alors les sentiers ouverts par l'écologie historique.

Travaillant sur l'environnement, l'historien devrait adopter les définitions de l'écologie puisqu'il utilise ses concepts, condition sine qua non pour avoir un langage commun. Ainsi de l'écosystème qui doit retrouver toute

\footnotetext{
20 Pichard, G., op. cit.
} 
sa dimension systémique, c'est-à-dire l'ensemble des interactions entre les éléments vivants et non vivants du milieu.

Cette définition de l'écosystème nécessite un jeu sur les échelles spatiales. Les limites de ces espaces sont variables, ce qui impose le recours à des chronologies fines : il faut là faire œuvre d'historien. L'attention doit être portée sur les articulations entre ces différents espaces et chronologies : il ne faut pas se contenter d'emboîtements. Il s'agit du premier niveau d'analyse avant la prise en compte des interactions pour atteindre la puissance heuristique de l'analyse systémique.

Nos travaux et la bibliographie brièvement rappelée ici permettent de prendre conscience des lacunes en écologie que comportent les démarches se réclamant de l'histoire de l'environnement et de celle de l'animal telles qu'elles sont faites par des historiens. Inversement, rares sont les travaux de biologie intégrant une réelle dimension historique des écosystèmes qu'ils étudient, c'est-à-dire une perception des mutations temporelles relevant du niveau d'analyse de l'historien. Certes, l'écologie aborde les agrosystèmes, les anthroposystèmes, dont les fondements sont indiscutablement humains et historiques. Cependant, bien souvent, elle prend plus en compte un état à un moment donné que l'épaisseur du temps, peut-être tout simplement du fait des domaines de compétence de la discipline et des chercheurs qui la font vivre. Par exemple, l'abandon de la notion de « forêt primaire » remplacée par le concept de «forêt ancienne », s'il donne toute son importance à la dimension historique de ce milieu, décrit un point de départ, non des processus politiques, sociaux et économiques. Ainsi, un chemin, plus ou moins escarpé, reste à parcourir par les deux disciplines.

Si l'histoire de l'environnement ne peut qu'être anthropocentrée, celle du loup - et des animaux en général ne peut pas se comprendre sans une transposition radicale du regard. Elle implique une double maîtrise : celle de l'écologie de l'espèce et celle des techniques d'accès, de manipulation et d'interprétation des données historiques. Plus généralement, une écologie historique qui n'est pas seulement une histoire de l'environnement de l'homme nécessite des compétences disciplinaires multiples.

Pour l'historien, demeure le questionnement sur les sources. Production humaine, elles ne rendent accessibles que les seuls écosystèmes anthropisés. Comment les interroger pour s'ouvrir à une approche systémique ? Cette réflexion ne peut se faire qu'une fois les éléments du système définis et leurs interactions caractérisées.
Pour l'écologue, tous les milieux ne sont pas accessibles, les hommes n'ayant pas généré partout et toujours des archives. D'autre part, l'historien ne peut pas être réduit au rôle de fournisseur de données sur "un écosystème disparu ». Ses outils ne se limitent pas à l'histoire quantitative créatrice de données chiffrées analysables par la méthode scientifique. Bien menée, l'analyse chiffrée conduit, d'elle-même, à poser de nouvelles questions qui trouvent leur place dans un contexte global dégagé par l'histoire.

\section{Conclusion}

Au terme de cet échange trop rapide entre un historien et un écologue, des avancées incontestables apparaissent. De l'histoire de l'environnement fatalement humain à l'écologie historique qui s'appuie sur une littérature déjà riche, du chemin a été parcouru. Le loup, la métairie et la forge : l'idée d'interactions entre milieu et société s'impose. Les regards se croisent. L'historien doit se convaincre de la nécessité d'une démarche systémique, quel que soit son objet d'étude ou sa problématique, surtout s'il se confronte à l'environnement. L'écologue, quant à lui, ne doit pas faire l'économie de l'histoire pour comprendre la genèse des milieux et des processus biologiques qu'il étudie. Il semble acquis que l'étude des écosystèmes anciens fait interagir des composantes sociales, économiques, techniques et écologiques. Dans un tel cadre épistémologique, l'histoire, tout en respectant ses spécificités, contribue à une synthèse où le point de vue de l'homme n'est plus le seul pris en compte. Mais, au-delà de la captation de vocabulaire, les rapports entre histoire et écologie restent encore incertains, leurs relations toujours ambiguës. L'environnement faussement «naturel», stricto sensu, est encore trop présent dans certains travaux, transformant les influences du milieu, qui sont indéniables, en un déterminisme fort. Deux règles de conduite s'imposent : élaborer un vocabulaire commun tout en restant attentif à son évolution dans la discipline qui le forge. Il devient alors possible de multiplier les études dans un dialogue permanent entre les deux disciplines.

\section{Remerciements}

Nous tenons à remercier Corinne Beck, historienne de l'environnement, pour les très profitables discussions sur, et autour, de ce texte. 